\title{
A Case Report on post Traumatic Angle Recession
}

\author{
Dr Nita Shanbhag ${ }^{1}$, Dr Bhaktee Walawalkar ${ }^{2}$ \\ ${ }^{I}$ Department Of Ophthalmology,Dr D.Y. Patil Medical College And Hospital,Nerul,Newmumbai, Dr DY Patil \\ University India. \\ ${ }^{I}$ Department Of Ophthalmology,Dr D.Y. Patil Medical College And Hospital,Nerul,Newmumbai,Dr DY Patil \\ University India.
}

\begin{abstract}
A 7 yr old boy presented with diminution of vision in left eye since 6 months, with an h/o ocular trauma 1 yr back.Patients history suggestive of some procedure done on the left eye after 10 days of trauma and inability to see, present clinical examination shows a paracentesis indicating an hyphema drainage procedure done in past,and a 0.9 cupping, suggestive of an acute rise of IOP immediate to the traumatic hyphema. Special investigations like anterior segment oct and ultrasound biomicroscopy suggestive of angle recession.

As the patient presented with intraocular pressure within normal range along with a significant cataract,a procedure of lens extraction along with posterior chamber intraocular lens implantation was carried out.This case should undergo regular follow ups for assessment of intraocular pressures and gonioscopy with serial fundus photographs for documentation of progression if any as these cases of angle recession, there are higher chances of progression to glaucoma.
\end{abstract}

Keywords: Angle recession,cataract, intraocular pressure, gonioscopy,glaucoma

\section{Introduction}

This a case report of a post traumatic angle recession with significant 0.9 cupping and an anterior subcapsular cataract at presentation in a $7 \mathrm{yr}$ old boy. Anterior segment OCT and Ultrasound biomicroscopy are good tools to demonstrate angle recession. Though intraocular pressures at presentation were within normal limits such cases should undergo a regular and long term follow up , as there are high chances of development of chronic glaucoma in these cases.

\section{Case history}

2.1 History of presenting illness: 7 yr old male presented to ophthalmology out patient department with history of gradual progressive diminution of vision in left eye(LE) since 6 months. No other ocular complaint at present.

2.2 Past history : Fire cracker injury to LE 1 year back, with an immediate painful red eye and diminution of vision.Patient gives history suggestive of an anterior chamber collection of blood and some procedure done on the left eye 10 days post trauma, exact history of management cannot be elicited, after which patient was asymptomatic for a few months, and later developed diminution of vision which was gradual, progressive ,painless and has reached the present state.

2.3 History of systemic illness : NIL

2.4 History of blood dyscrasis : NIL

2.5 History of congenital diseases : NIL

2.6 Family history : Insignificant

\section{Examination}

A detailed ocular examination of visual acuity, slit lamp biomicroscopy,Applanation tonometry,fundoscopy,and gonioscopy was done. Special Investigations like ultrasound biomicroscopy, Anterior segment OCT and Pachymetry was carried out . Patient did not cooperate for standard automated perimetry for assement of visual fields.

3.1 General examination : Patient is conscious, cooperative well oriented to time place and person.Vitals stable . 3.2 Ocular examination :

- Forehead and headposture : Normal

- No facial asymmetry. 


\begin{tabular}{|l|l|l|}
\hline & RIGHT EYE & LEFT EYE \\
\hline 1.Visual Acuity & $6 / 6$ & FC @ 1m \\
\hline 2.Slit lamp examination & Within normal limits & $\begin{array}{l}\text { Anterior subcapsular cataract } \\
\text { Mid dilated sluggishly reacting pupil. } \\
\text { 2 Paracentesis marks seen. }\end{array}$ \\
\hline 3.Fundus & $\begin{array}{l}\text { Media : clear. } \\
\text { Disc :0.6 cupping,well } \\
\text { defined margins, circular. } \\
\text { Macula and blood vessels : } \\
\text { Within normal limits } \\
\text { Foveal reflex }:+\end{array}$ & $\begin{array}{l}\text { Disc }: 0.9 \text { cupping with temporal disc } \\
\text { palour. } \\
\text { Macula and blood vessels : Within } \\
\text { normal limits } \\
\text { Foveal reflex }:+\end{array}$ \\
\hline 4.Applanation tonometry & $12 \mathrm{~mm}$ of hg. & $\begin{array}{l}\text { Omm of hg. } \\
\text { 5.Gen angles with angle recession } \\
\text { temporally. }\end{array}$ \\
\hline 6.Pachymetry & Open angles & 520 micron \\
\hline
\end{tabular}
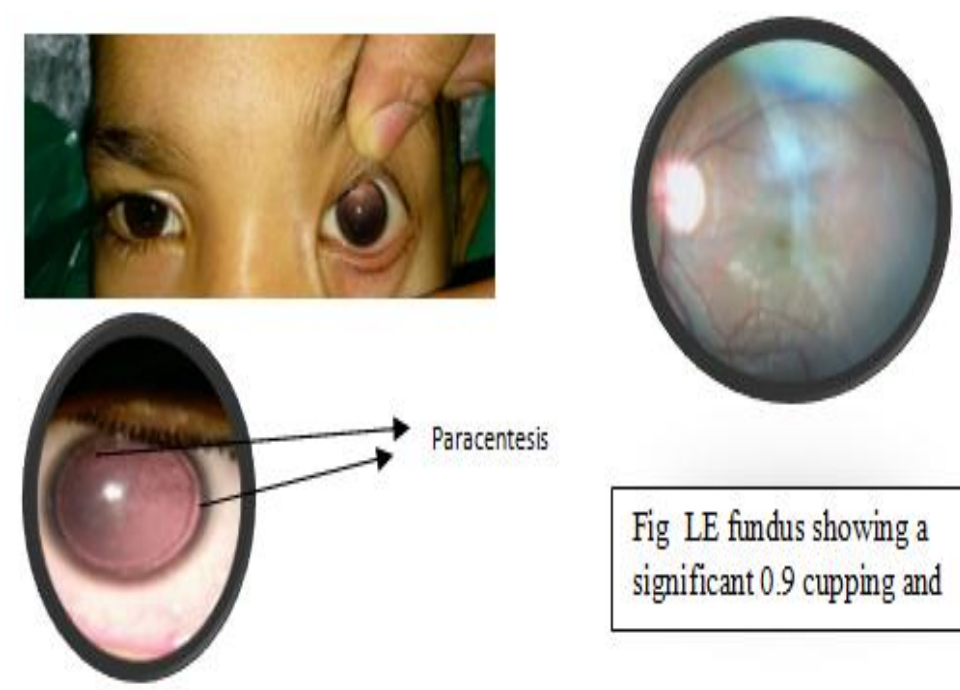

\section{Fig LE fundus showing a significant 0.9 cupping and}
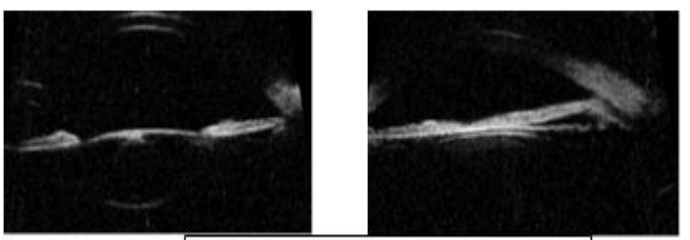

IMPRESSION: Left side large angle recession noted from 2 to 6.0 clock position. There is marked zonular stretching from 3 to 6.0 clock position.

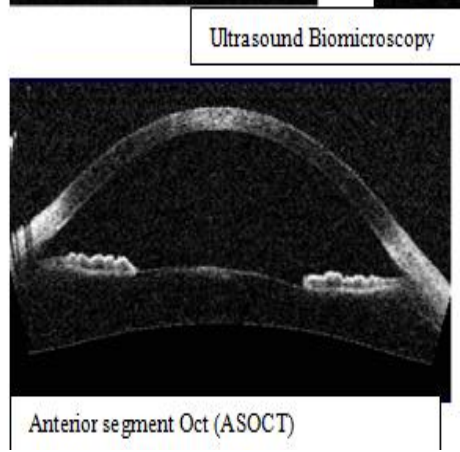

Both Anterior segment Oct (ASOCT) and Ultrasound biomicroscopy ( UBM ) are useful for cross-sectional imaging of the anterior segment and the AC angle. However in ASOCT structures behind the iris cannot be visualized where as in UBM the images of iris, ciliary body, zonules, peripheral lens, status of posterior capsule can be obtained. However UBM is more uncomfortable to the patient and requires a highly skilled operator, and provides a limited scan width. 


\begin{tabular}{|l|l|l|}
\hline & ASOCT & UBM \\
\hline Technology & Optical & Ultrasound \\
\hline Resolution & 15 microns & 50 microns \\
\hline $\begin{array}{l}\text { Longest Scan } \\
\text { Dimensions }\end{array}$ & $16 \times 16 \mathrm{Mm}$ & $5 \times 5 \mathrm{~mm}$ \\
\hline Contact With Eye & No & $\begin{array}{l}\text { Probe Does Not Contact } \\
\text { Eye Directly But } \\
\text { Requires Immersion } \\
\text { Bath }\end{array}$ \\
\hline Real Time Imaging & Yes & Yes \\
\hline Imaging Posterior To Iris & No & Yes \\
\hline $\begin{array}{l}\text { Quantitative } \\
\text { Measurement }\end{array}$ & Yes & Yes \\
\hline
\end{tabular}

\section{Management}

After eliciting the complete history and after all ocular examinations including slit lamp biomicroscopy, and special investigations like Anterior segment OCT and ultrasound biomicroscopy the condition was diagnosed as Anterior subcapsular cataract with angle recession. As it was a visually significant cataract along with intraocular pressures within normal range patient was posted for left eye lens extraction and Posterior chamber intraocular lens implantation under general anesthesia under guarded visual prognosis.

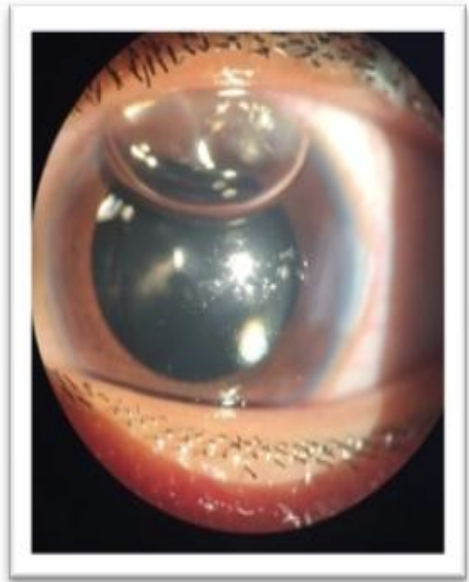

\begin{tabular}{|l|l|}
\hline & LEFT EYE \\
\hline Visual acuity unaided & $6 / 18$, improving to $6 / 9 \mathrm{p}$ \\
\hline Slit lamp examination & $\begin{array}{l}\text { Clear comea, clear comeal } \\
\text { incision seen temporally. } \\
\text { Well formed and quite AC } \\
\text { Posterior chamber intraocular } \\
\text { lens insitu, in bag. }\end{array}$ \\
\hline Applanation tonometry & \multicolumn{1}{|c|}{$16 \mathrm{~mm}$ of hg } \\
\hline Fundus & $\begin{array}{l}\text { Media : Clear } \\
\text { Disc :0.9 cupping with temporal disc } \\
\text { palour. } \\
\text { Macula and blood vessels : Within normal } \\
\text { limits } \\
\text { Foveal reflex : }+\end{array}$ \\
\hline
\end{tabular}

Fig . Post cataract surgery day 1

\section{Discussion}

Angle recession refers to a tear between circular and longitudinal fibers of ciliary body.Angle recession was first described by Collins in $1892^{1}$ In this case it has occurred as a sequelae to ocular trauma(fire cracker injury) along with an anterior subcapsular cataract. Anterior chamber hyphema causes outflow obstruction of trabecular meshwork, primarily with RBCs, however in some case pupillary block via a large clot may contribute to elevated IOP, causing a transient ischemic nerve damage which has lead to a significant 0.9 cupping .Girkin,etal. Used the US Eye injury Registry to demonstrate that 3.39\% of people go to develop angle recession glaucoma (ARG) at 6 months following blunt ocular trauma ${ }^{2}$. A 10 year prospective study of 31 eyes by Kaufmin andTolpin reported that $6 \%$ with angle recession will go on to develop glaucoma ${ }^{3}$.

Recession of greater than 180 degree associated with 4-9\% risk of incidence of glaucoma. and angles with greater than 240 degree are at highest risk of chronic glaucoma. ${ }^{4}$ Other risk factors for progression to glaucoma include, Chronically elevated IOP,poor initial visual aquity,lens injury and hyphema. ${ }^{5}$

Elevation of IOP from angle recession demonstrates a bimodal pattern, glaucoma occurs either within first yr or after $10 \mathrm{yrs}$ as described by Blanton.No racial predilection exist. No gender predilection exists.

\section{Conclusion}

As the patient had raised IOP post anterior chamber hyphema immediately post trauma,leading to a significant 0.9 cupping,this should be taken as a lesson and such cases of post traumatic hyphemas should be immediately taken up for drainage. An IOP of $25 \mathrm{mmhg}$ or more for 5 days with a total hyphema or an IOP of 60 
mmhg or more for 2 days are indications for surgery, former to prevent corneal blood staining and later to prevent optic atrophy ${ }^{6}$.Also any hyphema failing to resolve to less than $50 \%$ of the anterior chamber volume by 8 days despite medical management is also an indication due to peripheral anterior synechie formation. Indications of early intervention : sickle cell disease or sickle cell trait (IOP .25mmhg for $24 \mathrm{hrs}$ ), haemophilias, patients with existing glaucomas, children with more than half chamber hyphemas with risk of developimg amplyopia.

Multiple methods of surgical treatments have been suggested : Ac wash outs, clot removal via vitrectomy instrumentation or irrigation aspiration instrumentations and trabeculectomies with associated iridotimes have been carried out according to need, comfort and experience of the surgeon ${ }^{6}$ This patient had a delay in the intervention(10 days post trauma), possible reasons for delay in such cases can be delay in fitness for administration of General anesthesia, difficulty in monitoring IOP in a young child, patient on medical management ( e.g cycloplegics,systemic or tropical steroids,antifibrinolytics,analgesics,anti glaucoma medication )for a longer wait and watch period. As this patient presented with IOP within normal range, a plain cataract extraction for early visual rehabilitation and amblyopia prevention is carried out, this patient also has an angle recession component therefore he should undergo a regular yearly follow up for assessment of intraocular pressures and gonioscopy with serial fundus photographs for documentation of progression if any,as there is a documented high risk of development of chronic glaucomas in the long run .

\section{Acknowledgement}

None

\section{References}

[1]. Collins ET.On the phathological examination of three eyes lost from concussion chronic glaucoma.Trans Ophthalmol soc UK.1892;12:180-186

[2]. Girkin,C.,McGin,G.,Long,C., Morris,R., and Kunh,F.Glaucoma After OcularContusion:A Cohort Study of the United States Eye Registry.J Glaucoma.2005;14(6):470-3.

[3]. Kaufman J.Tolpin D.Glaucoma after traumatic angle recession.AM J Ophthalmol.1974;78:648-654

[4]. Alper MG.Contusion angle deformity and glaucoma.Arch Ophthalmol.1963;69;455-467.

[5]. Sihota R,Kumar S,Gupta V,et al.Early predictors of traumatic glaucoma after closed globe injurt:trabecular pigmentation, widened angle recess,and higher baseline intraocular pressure.Arch ophthalmol.2008 Jul.126(7):921-6.

[6]. Traumatic hyphema current stratergies;surgical therapy : www.aao.org 